\title{
Sentence Interpretation in Normal and Aphasic Hindi Speakers
}

\author{
JYOTSNA VAID \\ Texas A\&M University, College Station
}

AND

Rama PANDIT

All India Institute of Medical Sciences, New Delhi

\begin{abstract}
In interpreting a sentence, listeners rely on a variety of linguistic cues to assign grammatical roles such as agent and patient. The present study considered the relative ranking of three cues to agenthood (word order, noun animacy, and subject-vcrb agrecment) in normal and aphasic speakers of Hindi. Because animacy plays a grammatical role in Hindi (determining the nature and acceptability of sentences without accusative marking), this language is relevant to the claim that Broca's aphasia involves a dissociation between grammar and semantics. Results of Study 1 with normal Hindi-dominant speakers showed that animacy is the strongest cue in this language, while agreement is the weakest cue. In Study 2, Hindi-English bilinguals were tested in both their languages. Most showed the normal animacy-dominant monolingual pattern in Hindi, with a mixture of strategies from both languages in their interpretation of English. A substantial minority showed mixed strategies in both languages. Only 5 of 48 subjects displayed a complete separation between languages, with animacy dominance in Hindi and
\end{abstract}

This research was supported by a PHS-NIDCD grant on cross-linguistic studies of aphasia awarded to Elizabeth Bates and by a Council for International Exchange of Scholars IndoAmerican Advanced Research Fellowship awarded to Jyotsna Vaid. We acknowledge with gratitude the principals of St. Thomas School and Sophia Girls School in Meerut for allowing their students to participate in this study, and we thank Dr. Maharaj Singh of Meerut University for supervising data collection of the Hindi brain-intact subjects. Ms. Roxanne Samuelson of Speech Pathology in the ENT unit of the All India Institute of Medical Sciences, New Delhi, screened and tested the Hindi aphasic patients for this study. Miguel Quinones, John Williamson, Larry Juarez, and Virginia Marchman assisted in data analysis and prepared the graphs. A preliminary report of this research was presented at the ninth annual meeting of the South Asian Languages Analysis Roundtable, Cornell University, June 1987. Requests for reprints may be directed to the first author c/o Department of Psychology, Texas A\&M University, College Station, TX 77843-4235. 
word order dominance in English. In Study 3, two Hindi-English bilinguals with Broca's aphasia were tested in both languages. Results indicate (a) greater use of animacy in Hindi than in English and (b) greater usc of word order in English than in Hindi. The strategies displayed by these patients fall well within the range observed among bilingual normals. We conclude that the use of animacy in sentence interpretation by these aphasic patients reflects preservation of normal, language-specific processing strategies; it cannot be interpreted as a nonlinguistic strategy developed to compensate for receptive agrammatism. Results are discussed in light of other cross-linguistic evidence on sentence comprehension in monolingual and bilingual aphasics. (c) 1991 Academic Press, Inc.

In the process of sentence interpretation, listeners can rely on a variety of surface linguistic cues, including phonological stress, morphological markers, syntactic cues (especially word order), and semantic cues (e.g., noun animacy). The information conveyed by these cues allows listeners to identify the topic of a sentence, to distinguish between given and new information, and to assign grammatical roles. In acquiring a first or second language, a language user must learn what kinds of information are conveyed by the various linguistic cues present in the surface form of his language. The mapping between form and function that results from such a learning process is rarely one-to-one; instead, a single form can map to several functions and several forms can map to the same underlying function. For example, in English, cues such as word order, noun animacy, and case inflection on pronouns can all convey information about grammatical roles.

Within and across languages, individual cues can also vary in how well they convey information about sentence meaning. The accuracy with which a cue conveys information is known as its cue validity. Cue validity may be viewed as the product of a cue's availability and reliability and can be calculated from adult language input to the language learner (cf. McDonald, 1986, 1987).

\section{THE COMPETITION MODEL}

The concept of cue validity has been incorporated into a model of sentence processing developed by Brian MacWhinney and Elizabeth Bates. This model, known as the Competition Model (see Bates \& MacWhinney, 1982a; 1989), offers a probabilistic account of how cues combine during comprehension. In this model, each cue is accorded a strength or weighting proportional to its validity. This weighting in turn determines the amount of activation of a particular interpretation. If two cues point to the same interpretation, their strengths are combined (by an additive or a multiplicative rule-McDonald, 1986), leading to a greater activation of that interpretation compared to the activation produced by a single cue acting alone. If they disagree, the interpretation with the 
highest activation is chosen. In this way, cues cooperate and compete in the comprehension process.

The notion of cue validity and its psychological counterpart, cue strength, have been examined by MacWhinney, Bates, and Kliegl (1984) in a series of cross-linguistic experimental studies of sentence processing in children and adults and in brain-damaged individuals (Bates, Friederici, \& Wulfeck, 1987). The basic paradigm in these studies involves a sentence comprehension task in which native speakers of different languages are presented with simple, transitive sentences containing two concrete nouns and an action verb and are asked to identify the actor-that is, who performed the action described in the sentence. The sentences always represent some orthogonal combination of lexical-semantic, pragmatic, grammatical, and/or phonological cues. In some languages, the resulting list of stimuli includes a mixture of grammatical and semigrammatical sentences; in other experiments (including the experiment presented here), all the stimuli are ecologically valid. In some of these sentences the various cues converge to a single decision about who did the action; in other sentences, one or all of the cues may conflict. An example where the cues word order, subject-verb agreement, and animacy conflict is in the sentence "The dog are licking the pencils." Standard SVO word order in English would assign the preverbal noun "dog" as the actor, as would the animacy cue; but noun-verb agreement would favor the plural noun "pencils," which agrees in number with the plural verb form. In such cases, native speakers of English rely primarily on word order, thus discarding "pencils" as the actor even though it agrees with the verb. Italian speakers, however, are much more likely to choose "pencils" as the agent in this conflict situation; hence noun-verb agreement emerges as a stronger cue than word order in Italian. For German speakers, animacy and agreement are both stronger cues than word order, and for Hungarian and Serbo-Croatian speakers case is a stronger cue than word order. In short, normal adult listeners interpret sentences by making strong use of those cues that are most valid in their language. Thus, the relative ranking of cues will necessarily differ across native speakers of different languages, even when these languages (e.g., Italian and English) are typologically similar. (See MacWhinney \& Bates, 1989, for a summary of sentence comprehension studies in 13 different languages).

\section{SENTENCE INTERPRETATION IN NORMAL BILINGUALS}

The same sentence interpretation paradigm has also been applied in studies of bilingual adults (e.g., Bates \& MacWhinney, 1982b; Kilborn, 1989; Kilborn \& Cooreman, 1987; McDonald, 1986, 1987; Park, 1986; Vaid \& Chengappa, 1988; Wulfeck, Juarez, Bates, \& Kilborn, 1986; see Kilborn \& Ito, 1989, for a review). All of these studies demonstrate clear evidence for some kind of transfer or "leakage" of processing strategies 
from one language to another, regardless of the age at which a second language was acquired. The most common form of between-language interference is a clear transfer of strategies from Language 1 to Language 2 (e.g., overuse of subject-verb agreement and underuse of word order in the interpretation of English language sentences, by Dutch-English, Spanish-English, and German-English bilinguals). In other studies, individual adult bilinguals appear to have developed a single set of processing strategies that mixes the "best" cues from each language (e.g., a primary reliance on NVN in Spanish as well as English, with strong reliance on agreement and animacy on any sentence that deviates from NVN--see Wulfeck et al., 1986 for details). It is not yet clear what factors determine these variations in bilingual sentence processing. However, the existence of such mixed profiles in normal bilingual adults must constrain our interpretation of sentence interpretation by bilingual aphasics.

\section{SENTENCE INTERPRETATION AMONG APHASICS}

The language-specific differences in cue strength that we have just described appear to be preserved in individuals with language impairment following focal brain damage (Bates \& Wulfeck, 1989). For example, English-speaking aphasics, like normal English speakers, favor a firstnoun preference for NVN sentences, and a second-noun preference for VNN and NNV sentences. Italian-speaking aphasics, like normal Italian speakers, rely more on some combination of agreement and animacy to interpret sentences in their language. Furthermore, these cross-linguistic differences are preserved when we compare patients from the "same" clinical category. For example, English-speaking Broca's aphasics rely more on word order than Italian patients with the same clinical diagnosis; converscly, Italian-speaking Broca's aphasics makc more use of subjectverb agreement and animacy than their English counterparts. Nevertheless, there are some general trends in aphasic symptomatology that hold up across typologically distinct languages. Specifically, aphasic patients (fluent and nonfluent) display a marked reduction in the use of grammatical morphology compared with performance by normal controls in every language studied to date. This reduction is typically larger than the loss of sensitivity reported for any other cue type (i.e., word order, semantics), suggesting that some form of the closed-class hypothesis may generalize over languages.

The finding that semantic information is preserved in Broca's aphasia is certainly not new (e.g., Zurif \& Caramazza, 1976). However, there is still some controversy regarding the proper interpretation of this semantic sparing. In many of the original papers on "central agrammatism," the authors assume that semantics is preserved in agrammatic aphasia because it falls outside of the damaged grammatical processor. The cross-linguistic studies reviewed above suggest a different interpretation: degree of re- 
liance on semantic information is conditioned by language-specific grammatical facts (e.g., relative importance of word order and/or morphology in assignment of sentence roles); hence the degree of sparing or impairment that we see in use of semantic cues (e.g., animacy) will depend on the validity of those cues in the patient's premorbid language.

Information on sentence processing in normal and aphasic speakers of Hindi will be particularly informative in this regard, because the animacy contrast plays an important role within Hindi grammar, in determining the presence or absence of accusative marking on the object noun. In the present study, we will present data from normal, neurologically intact Hindi speakers and from two nonfluent aphasics who were fluent in both Hindi and English prior to their illness. We will use the same sentence interpretation paradigm described above, permitting us to compare this case study with a cross-linguistic large data base on sentence comprehension in monolinguals and bilinguals, normals and aphasics (see also Wulfeck et al., 1986; Vaid \& Chengappa, 1988).

Before we proceed, some remarks are in order about the characteristics of Hindi which are of relevance to this sentence interpretation task.

\section{SOME CHARACTERISTICS OF HINDI GRAMMAR}

Classification. Hindi belongs to the Indo-European language family. In fact, Hindi is a direct descendant of Sanskrit, the accepted source of all Indo-European language types.

Case. Hindi is a case-marked language, where case roles are marked by free-standing postposition markers (Khan, 1987). The nominative is a zero (unmarked) form; the accusative is marked through the addition of a single postposition "-ko," placed directly after the object noun phrase. (There is some controversy concerning the number and range of other case forms in Hindi, as discussed in Kellogg, 1938; Ganesan, 1975; and Bhatia, 1987; however, the remaining case contrasts need not concern us here).

In sentence interpretation studies performed on other case-marked languages (e.g., Serbo-Croatian in Smith \& Bates, 1987; Hungarian in MacWhinney, Pléh, \& Bates, 1985), case has emerged as a very strong cue to agent/object roles. Although detailed text counts of cue validity have not been conducted for Hindi, one would expect case to operate similarly; that is, one would expect case to emerge as the single strongest cue to sentence meaning. However, because one of our goals in this study was to compare Hindi and English, we restricted ourselves to cue types that are available in both these languages (e.g., word order, subject-verb agreement, and noun animacy). This was accomplished by using caseambiguous sentences with both nouns in the nominative form. Sentences of this type are perfectly grammatical and ecologically valid in Hindi (under conditions that are described in more detail below). However, as 
we shall see, this decision does have important consequences for the role of animacy in sentence interpretation.

Word order. The most common word order for simple sentences in the Hindi language is SOV, although many other word orders are possible under certain syntactic, morphological, and/or pragmatic conditions. Word order may serve as a cue to agenthood in both Hindi and English, but the validity of word order in Hindi is greatly reduced by the presence of these word order variations.

Subject-verb agreement. Hindi has an extensive system of agreement markers, including a set of subject-verb agreement contrasts that could scrve as cucs to sentence meaning. Subject marking on the verb involves contrasts for two levels of number (singular, plural), three levels of person (first, second, third), two levels of gender (masculine, feminine), and three levels of address (standard, intimate, respectful). Because there are so many agreement contrasts, we might expect subject-verb agreement to play an important role in Hindi sentence interpretation. However, there is also ample opportunity for ambiguity in the Hindi agreement system, particularly with third-person nouns.

Animacy/rationality. We have already noted that case-ambiguous sentences can occur in Hindi, with nominative case marking on both the subject and the object of a transitive verb. The conditions that permit omission of the accusative are quite complex; indeed, this is one of the most subtle and difficult aspects of Hindi grammar.

As described by Kellogg (1938, pp. 396-402), overt accusative marking is typically used when the object is a "rational being"; conversely, the nominative form is typically used for objects that are (a) irrational beings, (b) inanimate things, or (c) abstract terms. We should also note that accusative marking is not an inherent property of lexical items (universally provided for all animate but no inanimate nouns). Rather, the decision to us a "-ko" marker depends upon the way that the referent of a lexical item is construed by the speaker in a particular context. If the speaker wants to attribute rationality or willful intentions to an inanimate object, then provision of a "-ko" marker may be appropriate; in the same vein, omission of the "-ko" marker on an animate noun is also appropriate under certain circumstances, interacting with issues of definiteness and emphasis. For example, the "-ko" marker may be dropped when rational objects are given a generic meaning (illustrated by the sentence "You have killed children," used in well-known description of an historic massacre). This is roughly comparable to the contrast in English between "I want children" (a generic reference) and "I want the children" (a specific reference). Conversely, the "-ko" marker may be added to a definite irrational/inanimate object if the speaker wants to underscore its importance.

The nature of accusative marking in Hindi has three implications for 
the present study. First, it means that case-ambiguous sentences are perfectly grammatical in all the word order, animacy, and agreement conditions to be varied here. Second, it means that native Hindi speakers are biased to use the animacy contrasts provided in this experiment, because such contrasts play a major role in the interpretation of sentences without accusative marking. Third, the fact that animacy plays a central role within the grammar of Hindi raises interesting questions about the use of animacy by Broca's aphasics (i.e., patients with receptive and expressive processings that have been referred to collectively as "agrammatism"-Zurif \& Caramazza, 1976).

\section{STUDY 1: HINDI-DOMINANT NORMALS}

The aim of this study was to investigate the relative salience of word order, animacy, and noun-verb agreement in the comprehension of Hindi sentences by brain-intact, adult native users of Hindi.

\section{Method}

Subjects. A total of 57 female native Hindi speakers, ranging in age from 15 to 17 years, served as subjects for Study 1. All were students enrolled in the 12th grade of a convent school in Meerut, a city in northern India. Hindi was the first language for all of these students, and the language currently spoken in the home. It was also the language of instruction at school. However, bccause English is taught in the primary schools and widely used in movies and newspapers, in this city and elsewhere throughout India, these subjects cannot be viewed as pure monolinguals (i.e., individuals with no exposure to a second language). Indeed, pure monolinguals so defined are extremely rare in this country.

Stimuli. Stimuli consisted of simple, active, declarative Hindi sentences each containing two concrete nouns and a transitive action verb. All three word orders were tested (NVN, NNV, VNN) in orthogonal combinations of noun animacy (both nouns animate, first noun animate only, second noun animate only) and noun-verb agreement (ambiguous agreement where the verb agrees with both nouns, first-noun agreement only, or second-noun agreement only). This $3 \times 3 \times 3$ factorial design generated 27 sentences. Two exemplars of each sentence type were included, one using a singular verh and the other a plural verh, resulting in a total of 54 stimulus sentences. To minimize the effect of extralinguistic, real-world knowledge on sentence interpretation, the stimuli were prepared using a random assignment of animate and inanimate nouns to the designated slots. A list of the stimuli is available from the authors on request.

Procedure. The sentences were read out loud, one at a time in a neutral intonation by a native Hindi adult who was unaware of the theoretical rationale underlying the study. Upon hearing each sentence, subjects were to indicate which of the two nouns-the first or second-had performed the action described in the sentence. They were to write down the number 1 or 2 , corresponding to whether they thought the first or the second heard noun had performed the action.

Scoring. The dependent measure was choice of the first or second as the actor noun for each sentence. In coding the data, if the first noun mentioned was chosen, subjects were assigned a score of 1 . Choice of the second noun was assigned a score of 0 . Since there wcre two sentences with each possible combination of the three factors, the maximum score possible for any cell of the design was 2 , indicating that subjects chose the first noun both times. In presenting the data, we use percent choice of the first noun as agent. Thus, $100 \%$ 
TABLE 1

Summary of ANOVA Results of Normal Hindi Subjects $(N=57)$

\begin{tabular}{lccc}
\hline Effect & $d f$ & $F$ & $p<$ \\
\hline Animacy (An) & 2,112 & 85.46 & $.0001^{*}$ \\
Agreement (Ag) & 2,112 & 2.49 & .09 ns \\
Order (Or) & 2,112 & 7.99 & $.0006^{*}$ \\
An $\times$ Ag & 4,224 & 6.74 & $.0001^{*}$ \\
An $\times$ Or & 4,224 & 3.39 & $.01^{*}$ \\
Ag $\times$ Or & 4,224 & 6.59 & $.0001^{*}$ \\
An $\times$ Ag $\times$ Or & 8,448 & 3.83 & $.0002^{*}$ \\
\hline
\end{tabular}

would indicate a choice of the first noun on every item and $0 \%$ a choice of the second noun.

\section{Results and Discussion}

A $3 \times 3 \times 3$ repeated measures analysis of variance was performed on the dependent variable "percent choice of the first noun." This analysis (see Table 1) yielded a significant main effect of word order $[F(2,112)=$ $7.99, p<.0006]$ and of noun animacy $[F(2,112)=85.46, p<.0001]$ but no significant main effect of subject-verb agreement $(p<.09)$. The higher order interaction of all three variables was significant $[F(8,448)$ $=3.83, p<.0002]$, as were all the two-way interactions: Animacy $\times$ Agreement $[F(4,224)=6.74, p<.0001]$, Animacy $\times$ Order $[F(4,224)$ $=3.39, p<.01]$, and Agreement $\times \operatorname{Order}[F(4,224)=6.59, p<.0001]$.

Main effects. The animacy cue was very strong, accounting for more than $80 \%$ of the experimental variance (i.e., the variance under control of all the main effects and interactions taken together, regardless of significance level). Overall, when the first noun was animate and the second inanimate, percent choice of the first noun $(78 \%)$ was much greater than when both nouns were animate $(58.8 \%)$. Similarly, when the first noun of the sentence was inanimate and the second noun was animate, percent choice of the first noun was markedly reduced $(28.6 \%)$.

The next strongest cue for these native Hindi speakers was word order. Subjects showed a first-noun preference for assigning agenthood in sentences with a noun-verb-noun (NVN) order (58.2\%), consistent with, but not as strong as, the SVO preference shown by native speakers of English (e.g., Bates, McNew, MacWhinney, Devescovi, \& Smith, 1981; MacWhinney et al., 1984). Given that the canonical word order of Hindi sentences is subject-object-verb (SOV), the 59\% first-noun preference in the NNV order $(59.1 \%)$ indicates that subjects did in fact tend to interpret those sentences as SOV. The fact that they did not differentiate between the NVN and NNV sentences in their responses may reflect the less dominant role of word order in a case-marked language with extensive 


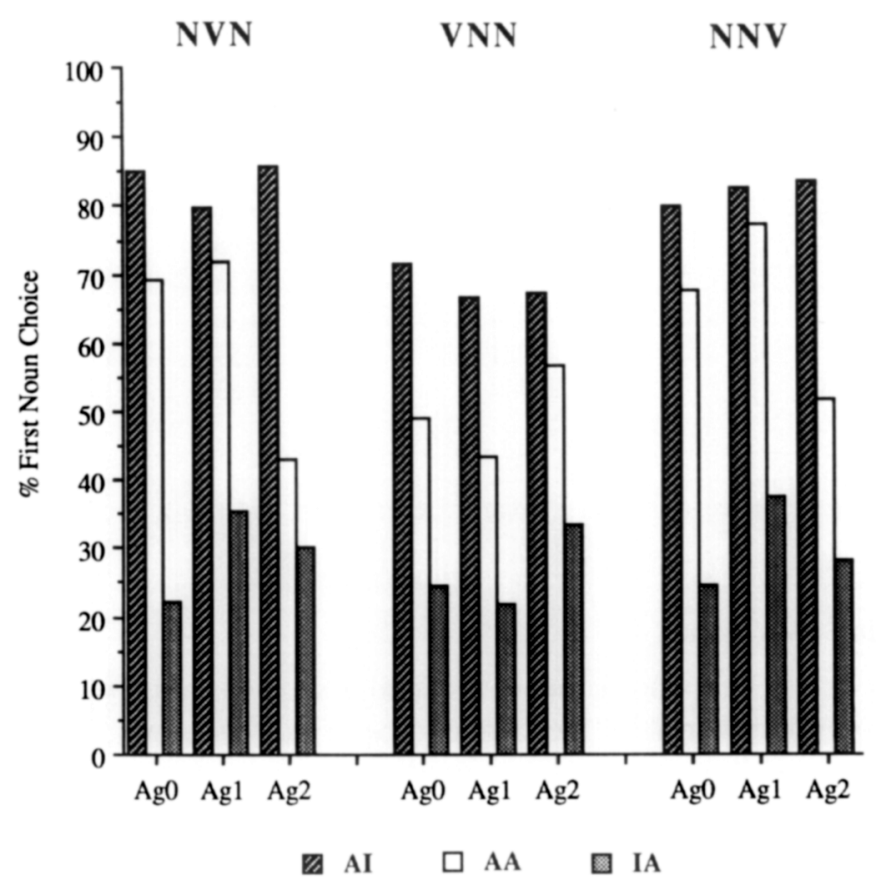

Fig. 1. Hindi monolinguals: Interaction of animacy, agreement, and word order.

word order variation. Subjects were close to chance performance (48.2\%) for sentences with a VNN order, a very small tendency toward VOS.

Subjects' overall performance on sentences varying in subject-verb number agreement was fairly similar across the three levels of the Agreement variable. When agreement cues were absent, the mean percent firstnoun choice was $54.8 \%$; when the verb agreed with the first noun, percent choice of first noun shifted slightly to $57.4 \%$ and dropped slightly to $53.2 \%$ when the second noun agreed with the verb. As noted above, this small difference between agreement conditions was not significant. The relative weakness of agreement cues in Hindi is somewhat surprising, given the large number of agreement contrasts that could serve as cues to sentence meaning in this language. Presumably, the tendency to ignore agreement in favor of animacy contrasts results from the fact that animacy plays a major role in the assignment (or omission) of accusative markers. Because there were no accusative markers on the sentences used in this experiment, native speakers appear to have invoked this language-specific constraint on the use of animacy to interpret agent/object roles.

Interaction effects. Given the significant three-way interaction, we need not consider the three significant two-way interactions. Figure 1 illustrates the three-way interaction of Animacy $\times$ Agreement $\times$ Order. 
To examine a complex interaction of this kind, we have found it useful to separate (1) sentences in which the dominant animacy cue is neutralized, and weaker cues can be evaluated on their own, (2) "coalition" sentences in which strong and weak cues point in the same direction, and (3) "competition" items in which strong or weak cues point in different directions.

First, it is clear that subject-verb agreement has a larger and more consistent effect on NVN and NNV sentences with no animacy contrast. For example, choice of the first noun as agent on NVN sentences is $72 \%$ when the verb agrees with the first noun versus $43 \%$ when the verb agrees with the second noun. Similarly, choice of the first noun on NNV sentences is $77 \%$ when agreement is with the first noun versus $52 \%$ when the verb agrees with the second noun. The relative weakness of agreement cues in Hindi is underscored by the fact that they do not "boost" decisions based on the dominant animacy cue. For example, choice of the first noun averages $85 \%$ in NVN-AI sentences with no agreement contrast; choice of first noun actually drops slightly (to $80 \%$ ) when the agreement cue is added (in NVN-AI-Ag1 items). Similarly, choice of the first noun averages $80 \%$ on NNV-AI items with no agreement contrast; addition of the agreement cues increases choice of the first noun by a mere $2.5 \%$ (on NNVAI-Ag1 items). Finally, the weakness of agreement contrasts in this experiment is made most evident by competition items (AI-Ag2 and IAAg1). For NVN sentences, percent first-noun choice was $35.5 \%$ for Ag1IA sentences compared to $80 \%$ for Ag1-AI sentences; the corresponding values for NNV sentences are $37 \%$ versus $82.5 \%$, and for VNN sentences, $22 \%$ versus $66.5 \%$. Hence animacy "wins" all competitions with subjectverb agreement.

We can use the same strategy to evaluate the role of word order in this experiment. Again, word order effects are most evident when animacy contrasts are missing and/or when animacy and word order point in the same direction (i.e., in coalition items). At the same time, word order strategies clearly "lose" when they are placed in competition with the animacy cue. For example, on NVN-Ag1-IA sentences where animacy contrasts with both agreement and word order, first-noun choice is still well under $40 \%$; similarly, on NNV-Ag1-IA items, our Hindi subjects chose the first noun less than $40 \%$ of the time. In other words, animacy "wins" even when it is pitted against a coalition of the two weaker cues.

Finally, an examination of semantically neutral AA sentences suggests that word order and subject-verb agreement are roughly equal in strength for these young control subjects. On NVN-AA-Ag2 items (where SVO and agreement compete), the first noun was chosen approximately $43 \%$ of the time (a very slight victory for agreement); on NNV-AA-Ag2 items (where SOV and agreement compete), first-noun choice averaged around $52 \%$ (a slight victory for the canonical SOV word order). 


\section{Summary}

This study was restricted entirely to Hindi sentences without accusative marking. Under these conditions, results for 57 young Hindi control subjects indicate that animacy is by far the strongest cue to agent/object roles. Subject-verb agreement and word order are both relatively weak; their effects are most evident when there is no semantic contrast at all and/or when animacy and these two "minor" grammatical cues converge on a common interpretation. Although these results may seem surprising to native speakers of English, they make sense when we remember that use of the accusative "-ko" marker depends upon subtle interactions with animacy, rationality, and definiteness. In other words, it may not be appropriate to treat animacy as an "extragrammatical" cue in the Hindi language. This result has interesting implications for the use of animacy by Hindi patients with Broca's aphasia.

\section{STUDY 2: BILINGUAL NORMALS}

Before we proceed to our two bilingual case studies, it is important to determine the extent to which this language-specific use of animacy is preserved in individuals who are daily users of both Hindi and English. Toward this end, English and Hindi versions of the stimuli used in Study 1 were administered to a sample of bilingual normals.

\section{Method}

Subjects. Participants in Study 2 were 48 young women from middle- to upper-class families in the northern Indian city of Meerut. All were 12th grade students at a convent school in this city, a school in which all instruction is carried out in English from the first grade onward. In addition to the forms of exposure to English experienced by the students in Study 1 (i.e., movies, newspapers, television), most of these students come from families in which English and Hindi are both used in the home (although Hindi is the first language acquired). Hence this is a sample of subjects who tend to use both languages on a daily basis.

Stimuli. Stimuli for the Hindi portion of Study 2 were identical to those employed in Study 1 . English stimuli were from the same pool of items, but not necessarily translations, and were equivalent to the stimuli employed by Bates, MacWhinney, and their colleagues in several studies of sentence interpretation by monolingual speakers of English (e.g., MacWhinney et al., 1984).

Procedure. We used the same procedure described for Study 1, above (i.e., oral presentation of stimuli with a written response). Half of the subjects $(N=24)$ received the Hindi version of the experiment first, with the English version administered at a separate session; the other half of the subjects $(N=24)$ received the English version first, followed by Hindi. Protocols were scored in the same manner reported for Study 1.

Design and analysis. Because testing was carried out in two languages, in counterbalanced orders, the design for Study 2 is necessarily more complex, a mixed design with one betweensubjects factor (order of presentation) and four within-subjects factors (Language, Animacy, Agreement, and Word Order). There were two levels of presentation order (Hindi first vs. English first), two levels of Language (Hindi vs. English), three levels of animacy (AnimateAnimate; Animate-Inanimate; Inanimate-Animate), three levels of agreement (Agreement 
TABLE 2

List of Significant Main Effects and Interactions in Study 2

\begin{tabular}{lrrl}
\hline Source & \multicolumn{1}{c}{$M S$} & \multicolumn{1}{c}{$F$} & \multicolumn{1}{c}{$p$} \\
\hline Language & 7.4541 & 10.49 & .002 \\
Presentation order & 4.5837 & 5.87 & \multicolumn{1}{c}{.02} \\
Animacy & 242.9008 & 250.83 & .0001 \\
Language $\times$ Animacy & 21.1323 & 41.33 & .0001 \\
Agreement & 14.3256 & 41.89 & .0001 \\
Language $\times$ Agreement & 4.6775 & 17.91 & .0001 \\
Presentation $\times$ Agreement & 1.8534 & 5.42 & .006 \\
Language $\times$ Presentation $\times$ Agreement & 1.7701 & 6.78 & .002 \\
Animacy $\times$ Agreement & 4.2481 & 14.86 & .0001 \\
Language $\times$ Animacy $\times$ Agreement & 3.6127 & 15.27 & .0001 \\
Word order & 129.8627 & 88.16 & .0001 \\
Language $\times$ Word order & 74.6914 & 102.69 & .0001 \\
Presentation $\times$ Word order & 9.9552 & 6.76 & .002 \\
Animacy $\times$ Word order & 3.8615 & 9.87 & .0001 \\
Language $\times$ Animacy $\times$ Word order & 1.5258 & 5.81 & .0001 \\
Presentation $\times$ Animacy $\times$ Word order & 1.5409 & 3.94 & .004 \\
Language $\times$ Presentation $\times$ Animacy & 1.0247 & 3.90 & .005 \\
$\quad \times$ Word order & & & \\
Agreement $\times$ Word order & 1.5658 & 8.28 & .0001 \\
Language $\times$ Animacy $\times$ Agreement $\times$ & .5895 & 3.11 & .002 \\
$\quad$ Word order & & & \\
\hline & & & \\
\hline
\end{tabular}

neutral; Agreement with the first noun; Agreement with the second noun), and three levels of word order (NVN, NNV, VNN). We will begin with results from the analysis of variance $(2 \times 2 \times 3 \times 3 \times 3)$. Then we will present an analysis of the strategies used by individual subjects (i.e., Hindi-dominant, English-dominant, and various mixtures of strategies from the two languages).

\section{Results and Discussion}

Analysis of Variance. A list of all the significant main effects and interactions is presented in Table 2. These include main effects of language, presentation order, animacy, and word order, plus a lengthy set of two-, three-, and four-way interactions. Although these results are quite complex, there were no real surprises; most of these effects follow the principles of convergence and competition described in other studies using this methodology (see MacWhinney \& Bates, 1989, for a summary). Furthermore, the Hindi results are similar in most respects to those observed in Study 1.

Starting with the main effect for language $[F(1,46)=10.49, p<.002]$, there was slightly more choice of the first noun overall in Hindi $(54 \%)$ than in English (49\%), reflecting a tendency for some subjects to follow the second-noun strategies (VOS and OSV) that have been documented in several different studies with English-speaking monolinguals and bi- 


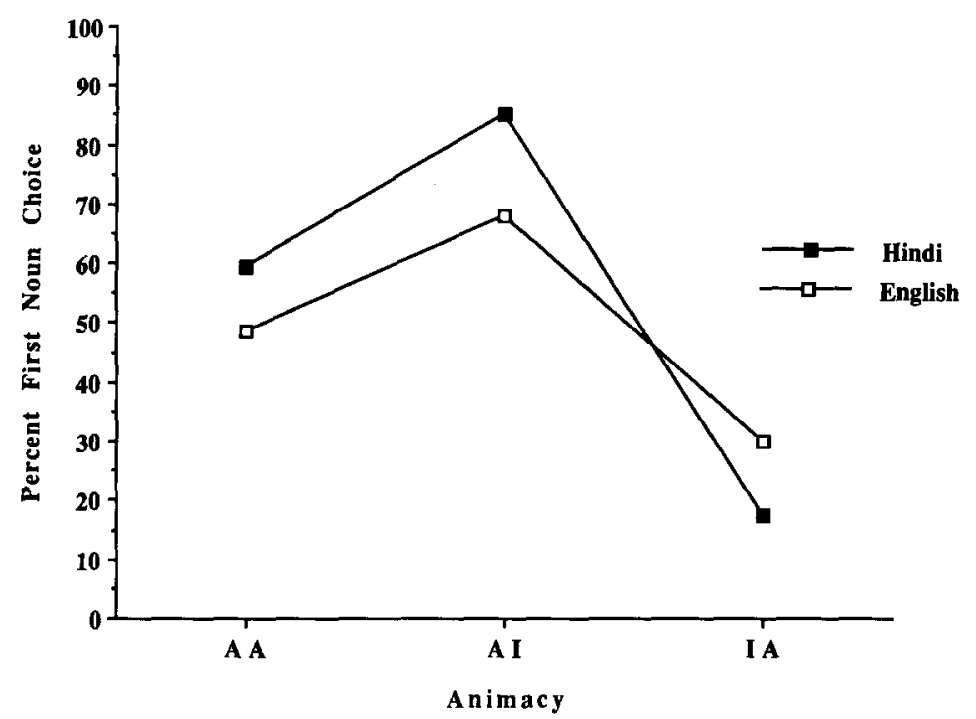

FIG. 2. Hindi-English bilingual normals: Interaction of language and animacy.

linguals. There was also a small but significant main effect of presentation order $[F(1,46)=5.87, p<.02]$, with slightly more choice of the first noun overall in subjects who were tested first in Hindi (53.5\%) compared with those who were tested first in English (49.4\%). In other words, more English-like patterns emerged overall when English was tested first. However, these language and presentation effects all interact with a number of other variables and must be interpreted in that light.

There was a very strong main effect of animacy $[F(2,92)=250.832$, $p<.0001$ ], by far the largest effect in the entire analysis. This effect was also in the predicted direction: greater choice of the first noun in AI sentences $(76.5 \%)$, much less first-noun choice on IA sentences $(23.8 \%)$, with a slight bias toward first-noun choice on semantically reversible AA items (54\%). However, there was also a significant interaction between animacy and language $[F(2,92)=41.33, p<.0001]$, with (as we might expect) larger animacy effects observed in Hindi (see Fig. 2). Note, however, that these bilingual subjects are still (as a group) applying animacy strategies to English to a much greater extent than we would expect for English monolinguals. In other words, there is solid evidence for transfer from L1 to L2. However, as we shall see, there are individual differences in the size and nature of these transfer effects.

The main effect of agreement is much smaller than the effect of animacy in this group of subjects, but (in contrast with Study 1) it is still reliable $[F(2,92)=41.89, p<.0001]$. As expected, the effect reflects greater choice of the first noun on Ag1 sentences (58\%), less on $\mathrm{Ag} 2$ sentences 


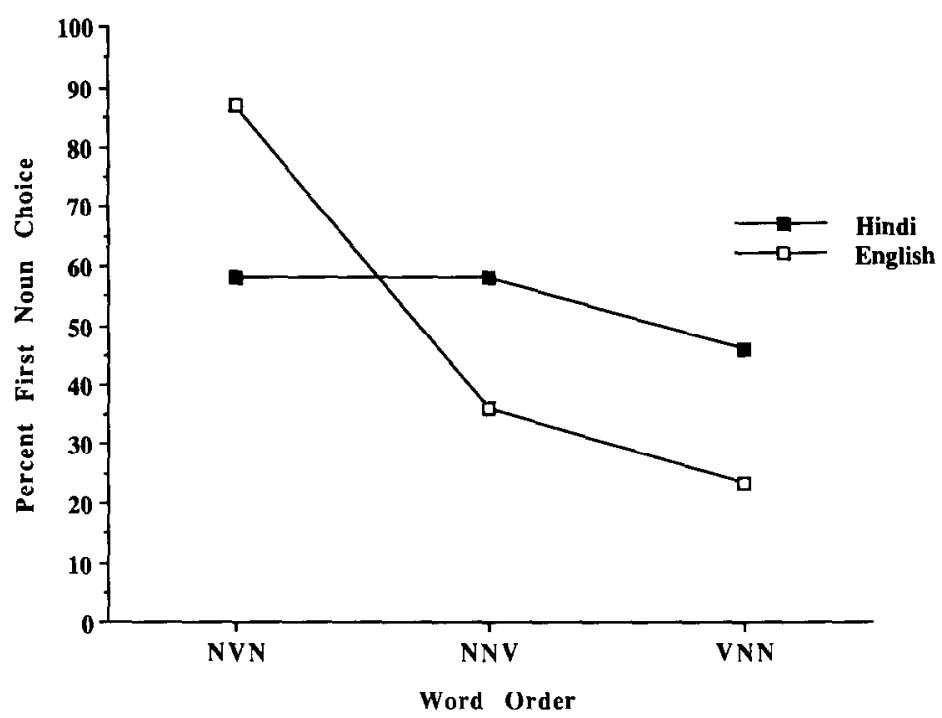

FIG. 3. Hindi-English bilingual normals: Interaction of language and word order.

(45\%), with morphologically neutral sentences falling in between $(51 \%)$. However, this effect also interacts with language (slightly larger morphology effects in Hindi-see Fig. 3) and with order of presentation (slightly smaller effects of agreement when Hindi is tested first). This presentation order effect is somewhat surprising (we might have expected initial testing in Hindi to enhance use of the agreement factor); nevertheless, the trend toward greater use of agreement in Hindi than in English holds within both groups, regardless of presentation order.

Collapsing over languages, the main effect of word order $[F(2,92)=$ $88.16, p<.0001]$ is larger than the effect observed in Study 1, but this fact must be interpreted in light of a very large interaction between language and word order $[F(2,92)=102.69, p<.0001]$. As illustrated in Fig. 4 , these results show a clear differentiation between languages at the group level. Overall, word order effects are larger in English and they are similar in direction (though not in size) to the word order results observed in several different studies of English monolinguals: a strong tendency to choose the first noun on NVN $(86.9 \%)$, with a tendency toward choice of the second noun on NNV (36.1\%) and VNN (23.4\%). By contrast, word order effects were relatively flat in Hindi: $58.3 \%$ on NVN, $54 \%$ on NNV, and (30.8\%). If we compare these Hindi word order patterns with the patterns observed in Study 1, we may conclude that there is some backward transfer from English in these bilingual subjects. For example, SVO is greater than SOV, a small but consistent reversal of the monolingual pattern, and the stronger VOS pattern observed in 


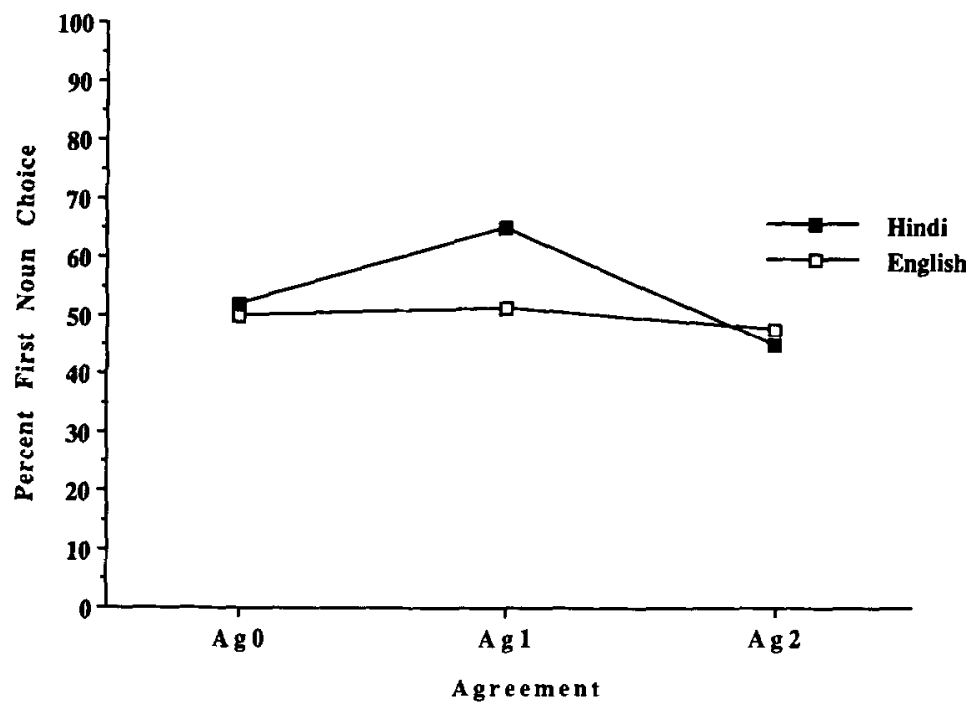

Frg. 4. Hindi-English bilingual normals: Interaction of language and agreement.

bilingual subjects might reflect a convergence between English (strong VOS) and Hindi (much weaker VOS).

In general, the higher order interactions in this analysis follow the convergence and competition predictions of the Competition Model, similar to the patterns described in Study 1. For example, agreement effects were strongest on semantically reversible items (i.e., where they do not have to compete with the dominant animacy cue). This was particularly clear when subjects were tested in Hindi (which accounts for the significant three-way interaction of language, animacy, and morphology). The higher order interactions involving language and word order follow a similar trend. For example, the English word order pattern (SVO, OSV, and VOS) emerges most strongly on English sentences that are semantically ambiguous. Similarly, the Hindi word order pattern (SVO and SOV) is also stronger on reversible AA items. These facts are responsible for the significant three-way interaction of language, animacy, and word order.

All of these interactions are affected somewhat by order of presentation. In most (although not all) cases, language-specific trends were enhanced in the language that was tested first (e.g., English-like word order patterns were particularly strong when English was tested first, especially on semantically reversible items; the Hindi word order pattern was strongest when Hindi was tested first, especially on semantically reversible items). However, in no case were the language-specific strategies that we have just described reversed or cancelled out as a function of order of presentation.

Overall, these results are compatible with a situation of language mixing. 
TABLE 3

Breakdown of Strategy Types Used by Hindi-English Bilinguals

\begin{tabular}{lccrr}
\hline & & \multicolumn{2}{c}{ Performance in hindi } & \\
\cline { 2 - 5 } & English-like & Mixed & Hindi-like & Total \\
\hline Performance in english & & & & \\
English-like & 0 & 4 & 5 & 9 \\
Mixed & 0 & 12 & 19 & 31 \\
Hindi-like & 0 & 17 & 31 & 8 \\
$\quad$ Total & 0 & & \\
\hline
\end{tabular}

That is, English effects appear to be affected by Hindi, and (to a lesser extent) Hindi effects are somewhat different in this sample of bilingual subjects compared with the Hindi monolinguals described in Study 1 (or, to be more precise, compared with native Hindi speakers with much less exposure to English). This leads us to a consideration of individual differences in language mixing and language transfer.

Analysis of individual strategies. The mix of strategies observed in these bilingual subjects is consistent with other comparative studies of bilinguals using this design (see Kilborn \& Ito, 1989, for a review), but there is somewhat more evidence for a separation of languages in this particular sample (i.e., more differentiation in the sentence interpretation strategies applied to Hindi vs. English stimuli).

Because animacy is the dominant cue in Hindi (at least for the caseambiguous sentences adopted here) while word order is the dominant cue in English, we based our analysis of individual strategies on the interaction of animacy and word order within cach subject. Subjects werc classificd as "Hindi-dominant" if they showed large effects of animacy and if animacy "wins" in all word order conditions. They were classified as "English-dominant" if they showed strong SVO, OSV, and VOS strategies and if all these strategies "win" in competition against animacy cues (e.g., preference for SVO in NVN-IA items, for OSV in NNV-AI items, and for VOS in VNN-AI items). They were classified as applying a mixed set of strategies if animacy won only a subset of the competitions against word order types (e.g., if they were animacy-dominant on NNV and VNN, but word order-dominant on NVN). This same classification procedure was applied separately within each language, for each subject. Hence an individual subject might be Hindi-dominant in both languages or Hindidominant in her native language with evidence for a mixture of strategies when tested in English and so on.

The breakdown of strategy types for each language is illustrated in Table 3. Of 48 subjects, we found only 5 who performed like monolinguals in each of their respective languages (i.e., English performance in English, 
TABLE 4

List of Mixed Strategy Types Used by Hindi-English Bilinguals

\begin{tabular}{lccr}
\hline Strategy type & English & Hindi & Total \\
\hline SVO > Animacy & 3 & 7 & 10 \\
SOV > Animacy & 0 & 2 & 2 \\
VOS > Animacy & 1 & 6 & 7 \\
(SVO + VOS) > Animacy & 17 & 1 & 18 \\
(SVO + SOV) > Animacy & 0 & 0 & 0 \\
SOV + VOS) > Animacy & 0 & 1 & 1 \\
(SVO + VOS + SOV) > Animacy & 1 & 0 & 1 \\
SVO = Animacy & 6 & 0 & 6 \\
Others & 3 & 0 & 3 \\
Total & 31 & 17 & 48 \\
\hline
\end{tabular}

Hindi performance in Hindi). The remainder show some evidence for transfer and/or blending of strategies between the two language types. The majority of these subjects (31) were Hindi-dominant when tested in Hindi. These include the 5 "double monolinguals" just described, another 7 subjects who displayed a pure Hindi pattern in both languages (i.e., total transfer), and 19 subjects who displayed a mixture of English and Hindi strategies when they were tested in English (i.e., partial transfer). Finally, another 17 subjects (roughly a third of the sample) display a mixed hierarchy of strategies for sentence processing in both languagesalthough in some cases the mixture was somewhat different in Hindi compared with English.

Table 4 lists the different mixture types (in English and/or Hindi testing), classified according to the different word orders that "won" over animacy. The most common by far (accounting for 17 cases in English testing and 1 case in Hindi testing) involved dominance of both SVO and VOS over animacy, with animacy winning only on NNV strings. This pattern makes sense if we remember that SVO and VOS are the preferred interpretations in both languages, while English and Hindi have opposite preferences for the interpretation of NNV (OSV and SOV, respectively). The next most frequent pattern was SVO dominance only, with animacy winning on the other two sentence types; this pattern accounted for 4 cases in English and 6 six cases in Hindi. Cases of SOV dominance were relatively rare, alone or in combination with SVO and/or VOS (3 cases in Hindi, 1 in English). In general, then, these mixed strategies tend to honor the strong word order types of English, particularly SVO and VOS (i.e., they are "English-driven").

To summarize the results of Study 2, there was considerable individual variation in the sentence interpretation strategies displayed by Hindi/ English bilinguals. For most subjects, results of Hindi testing mirror the 
results described in Study 1: a strong reliance on animacy, with weak agreement and word order biases that have their greatest effect when there is no strong semantic contrast. Most subjects showed a mixture of word order and animacy biases in their English testing, and some showed evidence of transfer in both directions (English $\rightarrow$ Hindi as well as Hindi $\rightarrow$ English). These intricate mixtures of animacy, word order, and subjectverb agreement can be used to argue in favor of the idea that animacy plays a grammatical role for Hindi-English bilinguals. Animacy cannot be viewed as a nonlinguistic heuristic that is applied when other cues fail. This conclusion has important consequences for the interpretation of results with bilingual Hindi-English Broca's aphasics.

\section{STUDY 3: BILINGUAL APHASICS}

To examine sentence comprehension under conditions of language breakdown produced by brain injury, the same sentence interpretation tests used in Studies 1 and 2 were given to two Broca's aphasics who were fluent in both Hindi and English prior to their illness. In previous studies applying this sentence interpretation paradigm to bilingual aphasics (Wulfeck et al., 1986; Vaid \& Chengappa, 1988), two trends have emerged. First, patients demonstrate some preservation of language-specific strategies for sentence interpretation, although (like normal bilinguals) they show considerable evidence for transfer or "leakage" between their two languages. Second, the bilingual patients tested to date demonstrate selectively greater retention of word order and/or animacy cues compared with subject-verb agreement-even in languages like Spanish, where agreement is ordinarily the dominant cue.

In the case study presented below, we are in a position to contrast animacy (the dominant strategy in Hindi) with word order (the dominant strategy in English). This comparison will permit us to distinguish between two views of semantic strategies in Broca's aphasia:

(1) Animacy strategies are nonlinguistic, applied by Broca's aphasics who have lost sensitivity to grammar-internal facts.

(2) Animacy strategies are language-specific, applied by Broca's aphasics in a fashion that reflects preservation of language-specific grammatical information.

If both our Hindi-English patients display a strong animacy bias in both their languages, it will be difficult to distinguish between these two hypotheses. On the one hand, an animacy-dominant result might well reflect loss of grammar and a corresponding reliance on nonlinguistic facts. On the other hand, because we know that many healthy bilinguals process both their languages with one dominant set of strategies, this configuration could mean that our two Hindi-English aphasics retain a Hindi-dominant mode of sentence interpretation. However, there are two other possible outcomes that would provide evidence in favor of Hypothesis 2. First, if 
word order dominates over animacy in one or both of these Hindi-English patients, then we would have further evidence in favor of the conclusion that some forms of grammatical information (particularly word order) are retained and used by Broca's aphasics. Second, if either or both of these patients display a selectively greater use of word order in English and a selectively greater use of animacy in Hindi, then we would have even stronger evidence in favor of overall preservation of grammar, and specific preservation of a grammar-internal use of animacy by Hindi patients.

\section{Method}

Subjects. Subjects were two upper-class male patients at the All-India Institute of Medical Sciences in New Delhi.

B.S. was a 39-year-old right-handed male, a homeopathic physician who suffered a CVA that left him with a right hemiparesis. A CT scan showed evidence of an acute infarct throughout the territory of the left middle cerebral artery. B.S. was classified as a Broca's aphasic following a battery of clinical tests used at the All India Institute of Medical Sciences. His first languages were Hindi and Punjabi, although he learned English early in school. In response to questions about his premorbid language use, he reported use of Hindi and Punjabi at home, all three languages with friends, English only at work, and Hindi and English to read newspapers and write letters. This patient was tested in Hindi first, followed by English.

B.G. was a 71-year-old right-handed male who suffered a CVA that left him a transient right hemiparesis. CT scan was inconclusive, but he continued to suffer a right facial weakness that persisted at the time of testing. Although we have no information on his education or occupation prior to his illness, B.G. comes from a relatively wealthy family in northern India (e.g., he was sent for medical consultation to London and New York following the CVA). After clinical examination at the All India Institute of Medical Sciences, he was classificd as a Broca's aphasic (i.e., severe expressive language deficits with relative sparing of comprehension at a clinical level). B.G.'s first spoken languages were Hindi/Urdu and Punjabi; he learned English in school. A brief interview concerning his premorbid profile of language use suggests that he used Punjabi and Hindi at home and all three languages at work and with friends. He used English to read newspapers, and wrote letters in English and Urdu (an Arabic script which corresponds on the spoken level to Hindi). B.G. was tested in English first, followed by Hindi.

Stimuli and procedure. The same set of sentences used in Study 2 were administered in the present experiment. Sentences were read aloud to the patients by a research assistant, a nurse in the Speech Pathology unit of the Medical Institute, whose duties included obtaining background information on the patients. Patients signaled their responses by pointing to the appropriate noun on the stimulus sheet. Bilinguals were tested in both their languages on separate days.

The data were coded as in Studies 1 and 2, except that a score of 0.5 was assigned to any trials on which subjects did not respond at all.

\section{Results}

Given the small sample size, we did not apply inferential statistics to these data. Instead, we will provide each patient's mean percent first choice score for each of the three main variables (animacy, agreement, word order) in each language (Hindi vs. English).

Figure 5 illustrates the animacy strategies for each patient (collapsing 


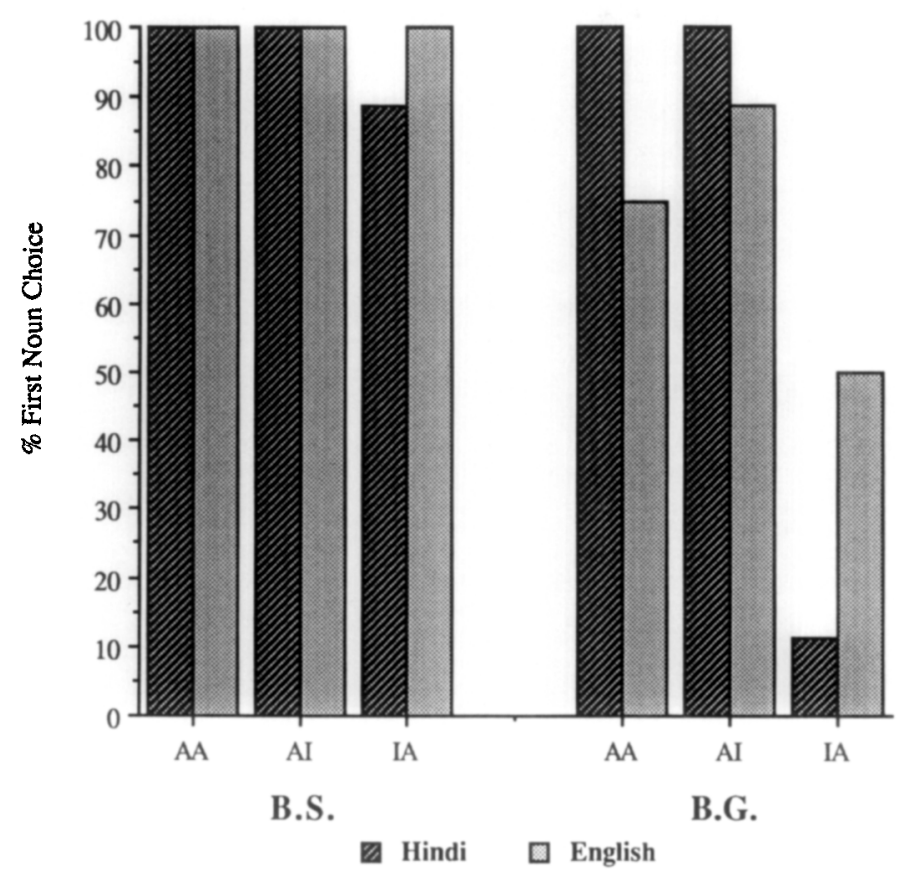

Fig. 5. Hindi-English bilingual aphasics: Effects of animacy in each language.

across word order and agreement contrasts). From this figure it is clear that patient B.S. adopted a massive word order strategy for almost all sentence stimuli in this experiment. He chose the first noun $100 \%$ of the time on Animate-Animate and Animate-Inanimate items; choice of the first noun dropped to $89 \%$ in Hindi (representing 2 second-noun choices of 18 possibilities), but stayed at $100 \%$ in English. By contrast, Patient B.G. clearly retained the animacy strategy that is characteristic of monolingual Hindi speakers and most Hindi-English bilinguals tested in Hindi. Furthermore, he applied the animacy strategy to a much greater extent in Hindi compared with English. On Hindi items, choice of the first noun was $100 \%$ for $\mathrm{AA}$ and $\mathrm{AI}$ sentences, but only $11 \%$ for items in which the first noun was inanimate (IA); on English items, the corresponding figures are $75 \%$ (AA), $89 \%$ (AI), and $50 \%$ (IA). In other words, B.G. applied the animacy strategy differentially in his two languages, relying more on animacy when he was tested in Hindi.

Figure 6 demonstrates the word order effects (collapsing over animacy and agreement) for each patient in Hindi and English, respectively. Again, it is clear that patient B.S. relied overwhelmingly on word order information, a first-noun strategy that is applied to all word order types in Hindi and English. Patient B.G. showed a very different configuration. 


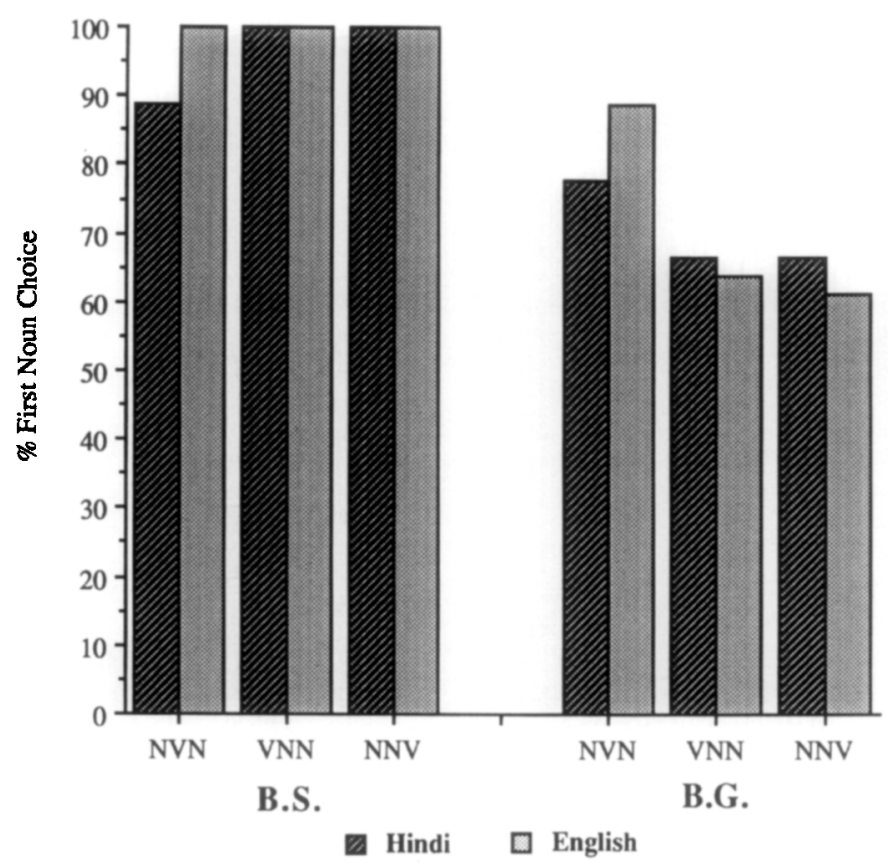

Fig. 6. Hindi-English bilingual aphasics: Effects of word order in each language.

In both languages, SVO was the dominant word order, although this strategy was stronger in English (89\%) than it was in Hindi (78\%). Firstnoun choice was smaller on VNN and NNV, particularly in English. Once again, B.G. showed some differentiation between his two languages, with more consistent use of word order (particularly SVO) when he was tested in English.

Finally, Fig. 7 illustrates the subject-verb agreement effect for these two patients (collapsing across animacy and word order). Again, Patient B.S. ignored subject-verb agreement in favor of his across-the-board firstnoun strategy. Patient B.G. showed no consistent ability to use subjectverb information. Choice of the first noun was slightly higher on Ag1 sentences (agreement on the first noun) compared with items that are morphologically ambiguous; however, choice of the first noun is also higher on Ag2 sentences compared (again) with items that have no subject-verb agreement contrast. In other words, although his behavior is somewhat affected by the presence of an agreement cue, he appears to be insensitive to the direction of that cue. The disappearance of subjectverb agreement in these two patients is not surprising, since it would be predicted on two grounds: (a) the relative weakness of agreement cues among normal controls in Hindi (Study 1) as well as English (MacWhinney et al., 1984; Study 2) and (b) the finding that grammatical morphology 


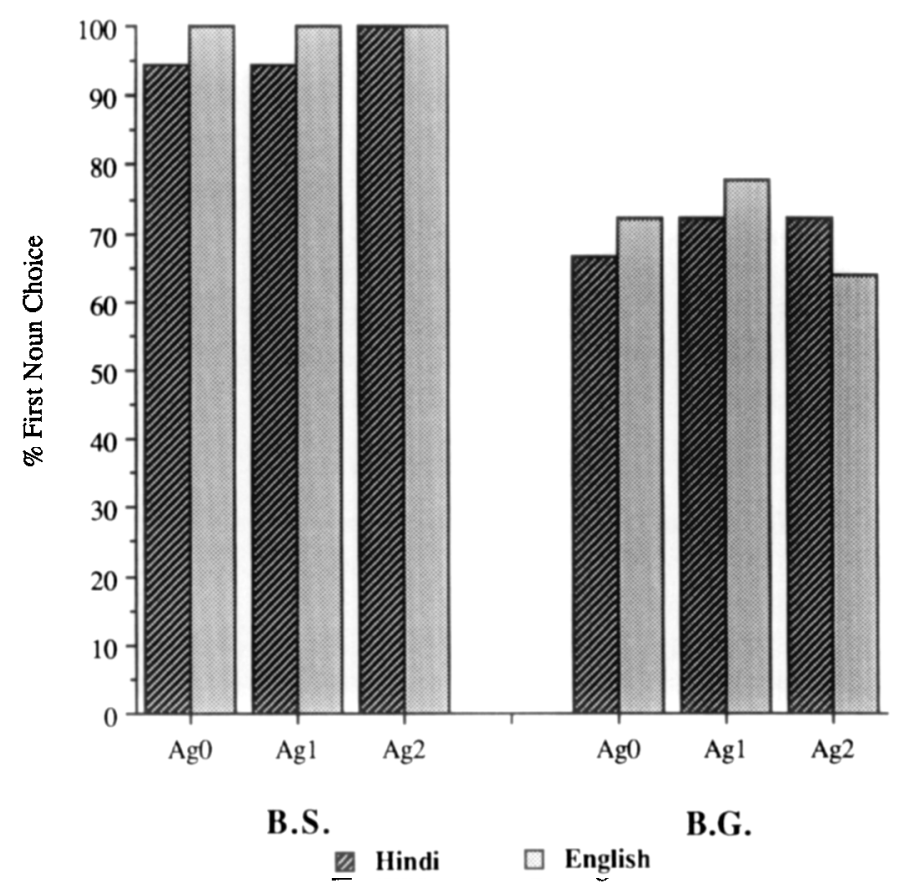

FIG. 7. Hindi-English bilingual aphasics: Effects of agreement in each language.

tends to erode in aphasic patients in all the languages that have been studied to date using this paradigm (Bates \& Wulfeck, 1989).

\section{Summary}

The sentence interpretation profiles displayed by these two Hindi-English patients add to a growing body of evidence suggesting that crosslinguistic differences in sentence interpretation are preserved in Broca's aphasia. Patient B.S. displayed an unusual profile in both his languages, applying an across-the-board strategy of choosing the first noun as agent. However, he did make two exceptions in Hindi, for Inanimate-Animate sentences that are widely interpreted as OVS by Hindi normals. Patient B.G. relied instead on a strong animacy strategy, interacting with a weaker effect of SVO; however, he used animacy to a lesser degree (and SVO word order to a greater degree) in the interpretation of English sentences.

We noted in Study 2 that normal bilingual adults show substantial variation in their sentence-interpretation strategies, ranging from complete dominance of Hindi over English (with little or no differentiation between the two languages) to a mixed hierarchy of strategies with features from both languages (e.g., SVO dominance on NVN strings, animacy dominance on NNV and VNN). Patient B.G. falls into the latter type. His 
pattern is consistent with findings by Wulfeck et al. (1986) for one SpanishEnglish bilingual; but it is also consistent with the performance displayed by several of our normal bilingual subjects in Study 2. By contrast, the array of first-noun strategies used by Patient B.S. (SVO, VSO, and SOV) does not resemble any of the mixed options uncovered in Study 2 (Table 4). Instead, it bears more resemblance to the overuse of canonical word order by monolingual German-speaking Broca's and Wernicke's aphasics (Bates et al. 1987) and may reflect the specific effects of focal brain injury on a patient who also displayed a mixed hierarchy of strategies (with NVN dominant over animacy) in his premorbid state.

\section{CONCLUSION}

The Hindi language offers a special perspective on the study of sentence processing in normals and aphasics because of the interesting interactions between grammatical and semantic factors in this language. Specifically, the provision or omission of accusative markers is conditioned by intricate interactions with animacy, rationality, and/or definiteness. Results from Study 1 suggest that normal young Hindi speakers rely overwhelmingly on the animacy contrast to interpret case-ambiguous sentence stimuli, with significant but weak contributions by word order and subject-verb agreement that are most apparent on semantically reversible AnimateAnimate sentence types. This behavior is in line with the nature of case marking in Hindi: by omitting accusative markers from these stimuli, we placed our listeners in a situation in which animacy contrasts take on paramount importance.

Performance by 48 Hindi-English bilinguals confirms this general picture. The majority of these bilingual subjects showed the same animacydominant strategy when tested in Hindi (replicating Study 1). However, there was also considerable evidence for language transfer and language mixing, especially in English testing. The mixed strategies almost all involved an interpolation of word order and animacy strategies, suggesting that these two sources of information can be integrated on an equal footing. Furthermore, most subjects used a different mix of strategies in Hindi vs. English, further evidence that animacy is treated as a languagespecific cue (as opposed to a nonlinguistic heuristic developed for use in this experiment).

Because animacy plays a language-specific and perhaps a grammarinternal role in the Hindi language, the differential performance displayed by two bilingual Hindi-English Broca's aphasics can be used to argue against the idea that semantic strategies are "nonlinguistic" or "extragrammatical" when they are applied by aphasic patients. One of our two patients (B.S.) relied much more on word order than animacy in both of his languages (although animacy was somewhat more important in Hindi). Such overreliance on word order has been reported before and it is not 
entirely surprising in a patient who was once quite fluent in English; however, it is interesting that he preferred word order to a semantic alternative. Our other patient (B.G.) showed a mixture of Hindi and English strategies, with somewhat more use of animacy for Hindi sentences and more use of word order for English sentences. This partial differentiation between English and Hindi is within the range that has been observed in studies of normal bilinguals.

In future studies, we plan to pursue a broader range of interactions among case, animacy, rationality, and definiteness in Hindi and related languages (see also Vaid \& Chengappa, 1988). We are convinced that the use of animacy is linguistically conditioned in these Hindi-speaking patients. However, it remains to be seen whether or to what extent Hindispeaking aphasics retain sensitivity to the subtle range of pragmatic and semantic factors that control overt case marking in this language.

\section{REFERENCES}

Bates, E., Friederici, A., \& Wulfeck, B. 1987. Comprehension in aphasia: A cross-linguistic study. Brain and Language, 32(1), 19-67.

Bates, E., \& MacWhinney, B. 1982a. Functionalist approaches to grammar. In E. Wanner \& L. R. Gleitman (Eds.), Language acquisition: The state of the art. New York: Cambridge Univ. Press.

Bates, E., \& MacWhinney, B. 1982b. Second language learning from a functionalist perspective: Pragmatic, semantic and perceptual strategies. In H. Winitz (Ed.), Foreign and native language acquisition: Annals of the New York Academy of Sciences. New York: New York Academy of Sciences. Pp. 190-214.

Bates, E., \& MacWhinney, B. 1989. Functionalism and the competition model. In B. MacWhinney \& E. Bates (Eds.), The crosslinguistic study of sentence processing. New York: Cambridge Univ. Press.

Bates, E., McNew, S., MacWhinney, B., Devescovi, A., \& Smith, S. 1981. Functional constraints on sentence comprehension: A cross-linguistic study. Cognition, 11, 245299.

Bates, E., \& Wulfeck, B. 1989. Cross-linguistic studies of aphasia. In B. MacWhinney \& E. Bates (Eds.), The crosslinguistic study of sentence processing. New York: Cambridge Univ. Press.

Bhatia, T. 1987. A history of the Hindi grammatical tradition: Hindi-Hindustani grammar, grammarians, history and problems. New York: Brill.

Ganesan, S. 1975. A contrastive grammar of Hindi and Tamil. Chepauk, Madras: Univ. of Madras Press.

Kellogg, S. 1938. A grammar of the Hindi language. London: Routledge \& Kegan Paul. Third ed.

Kilborn, K. 1989. Sentence processing in a second language: The timing of transfer. Language and Speech, 32, 1-23.

Kilborn, K., \& Cooreman, A. 1987. Sentence interpretation strategies in adult DutchEnglish bilinguals. Applied Psycholinguistics, 8, 415-431.

Kilborn, K., \& Ito, T. 1989. Sentence processing strategies in adult bilinguals. In B. MacWhinney \& E. Bates (Eds.), The crosslinguistic study of sentence processing. New York: Cambridge Univ. Press.

Khan, B. 1987. The ergative case in Hindi-Urdu. Studies in the Linguistic Sciences, 17(1), 91-101. 
McDonald, J. 1986. Assigning linguistic roles: The influence of conflicting cues. Journal of Memory and Language, 25, 415-431.

McDonald, J. 1987. Sentence interpretation in bilingual speakers of Dutch and English. Applied Psycholinguistics, 8, 379-413.

MacWhinney, B., \& Bates, E. (Eds.) 1989. The crosslinguistic study of sentence processing. New York: Cambridge Univ. Press.

MacWhinney, B., Bates, E., \& Kliegl, R. 1984. Cue validity and sentence interpretation in English, German and Italian. Journal of Verbal Learning and Verbal Behavior, 23, 127-150.

MacWhinney, B., Pléh, C., \& Bates, E. 1985. The development of sentence interpretation in Hungarian. Cognitive Psychology, 17, 178-209.

Park, K. S. 1986. Sentence interpretation in Korean-English bilinguals. Unpublished manuscript, Texas A\&M University, College Station, TX.

Smith, S., \& Bates, E. 1987. Accessibility of case and gender contrasts for agent-object assignment in Broca's aphasics and fluent anomics. Brain and Language, 30, 8-32.

Vaid, J., \& Chengappa, S. 1988. Assigning linguistic roles: Sentence interpretation in normal and aphasic Kannada-English bilinguals. Journal of Neurolinguistics, 3(2), 161-183.

Wulfeck, B., Juarez, L., Bates, E., \& Kilborn, K. 1986. Sentence interpretation strategies in healthy and aphasic bilingual adults. In J. Vaid (Ed.), Language processing in bilinguals. Hillsdale, NJ: Erlbaum.

Zurif, E., \& Caramazza, A. 1976. Psycholinguistic structures in aphasia: Studies in syntax and semantics. In H. Whitaker \& H. A. Whitaker (Eds.), Studies in neurolinguistics New York: Academic Press. Vol. 1. 